\title{
Lipostatic control of body weight: Evidence of humoral mediation
}

\author{
KENNETH R. KING \\ State University of New York at Buffalo, Buffalo, New York 14214
}

\begin{abstract}
Pairs of rats were implanted with chronic venous cannulae and cross-perfused in order to investigate the role of humoral factors in the regulation of body weight. Five-hour-deprived rats were cross-perfused with obese, normal weight, or lean partners on each of 4 successive days. Relative to the control condition (normal-weight partner), animals with obese partners consumed less and those with lean partners consumed more food immediately after blood-mixing. The results suggest that a humoral factor, related to total body fat, is involved in the long-term regulation of body weight. No conclusions are drawn as to the chemical nature of the factor or the mechanism by which intake is altered, but possible involvement of palatibility factors is discussed.
\end{abstract}

For any theory attempting to explain how central mechanisms control food intake, it is necessary to explain the manner in which the brain is informed about the nutritional state of the body. According to the glucostatic (Mayer, 1953) and the lipostatic (Kennedy, 1953) theories, humoral changes related to sugar or fat metabolism are detected by the hypothalamus, which in turn modifies subsequent behavior. While the glucostatic theory holds that hunger and satiety are due primarily to short-term shifts in blood glucose levels (or utilization rates), the lipostatic hypothesis states that the humoral signal which influences central structures must be related to long-term changes in the adipose tissue stores.

Evidence for humoral involvement in short-term control comes from the finding by Davis, Gallagher, and Ladove (1967) that deprived rats, cross-perfused with satiated partners, consumed $50 \%$ less food than under similar conditions prior to blood-mixing. Since deprived animals which were cross-perfused with other deprived rats did not show this reduction in intake, Davis and his colleagues concluded that the blood from satiated rats must contain a "satiety" factor capable of inhibiting food intake even in hungry animals. Similar results were found in sheep (Seoane, Baile, \& Martin, 1972), but in a study using rhesus monkeys (Walike \& Smith, 1972) no evidence of humoral factors relating to hunger and satiety was found.

Humoral factors may also play a role in the longterm regulation of body weight. Recent findings indicate that the brain may regulate weight about some central "set point" by utilizing feedback from the periphery. Artificial manipulations of an animal's weight have been shown to result in adjustments in

The author's current address is Psychology Department, Allegheny College, Meadville, Pennsylvania 16335. its food intake sufficient to restore weight to a previous set-point level (Hoebel \& Teitelbaum, 1966; Powley \& Keesey, 1970). These findings strongly suggest the existence of a feedback system for maintaining body weight constant. In addressing this issue, Liebelt, Bordelon, and Liebelt (1973) have proposed that the anatomically dispersed fat organs of the body may act as a unitary organ complex capable of exercising a form of autoregulation through humoral communication with the brain. Evidence that body fat may actually be responsible for the formation of a humoral feedback signal is provided by the classic study by Hervey (1959). In this, he showed that normal animals, parabiotically joined to partners made hyperphagic and obese by ventromedial hypothalamic lesions, gradually stop eating and die of starvation. Although others have failed to replicate this finding (Han, Mu, \& Lepkovsky, 1963), it remains a positive indication that there may be a humoral factor, covarying with body adiposity, which can inhibit food intake in normal animals.

Should such a factor exist, it might be expected to vary either directly or conversely with total adipose tissue. Assuming that the factor's concentration would be correlated with the size of the fat stores, one might predict that blood from animals with excesses or deficiencies of fat would cause changes in food intake when infused into normal animals. Presumably, blood from obese rats would decrease intake while blood from lean rats would augment intake. The purpose of this experiment, then, is to utilize Davis' cross-perfusion technique to investigate the possible roles of humoral-factors in the long-term regulation of body weight.

\section{METHOD}

\section{Subjects}

The subjects were 60 male Sprague-Dawley rats (ARS Sprague- 
Dawley, Madison, Wisconsin) between 90 and 110 days old. All animals were individually housed in a constant-temperature room $\left(22^{\circ} \mathrm{C}\right)$ and provided with constant food and water. Lights were set automatically to go off at $6: 00 \mathrm{p} . \mathrm{m}$. and to come on at 4:00 a.m.

\section{Apparatus}

The cannulae that were implanted into the external jugular veins of the animals were similar to those described by Davis, Gallagher, Ladove, and Turausky (1969). Basically, their construction involved joining a short $(4-\mathrm{cm})$ segment of silicone tubing (Silastic Medical Grade No. 602-155, i.d., = .025 in.) to a longer $(20-\mathrm{cm})$ segment of polyethylene tubing (Intramedic P.E. 60 , i.d. $=.030$ in.). While one end of the Silastic segment was being softened by immersion in trichloroethylene, one end of the polyethylene tubing was flared with the heat of a match. The softened silicone tubing was then slipped over the flared polyethylene to form a tight junction as the solvent evaporated and the silicone returned to its normal resiliency. The Silastic segment was then snipped off at a $45^{\circ}$ angle with scissors about $3 \mathrm{~cm}$ from this junction. The other end of the polyethylene was then wrapped around a pencil six or seven times and plunged into hot $\left(90^{\circ} \mathrm{C}\right)$ water to form the tubing into a shape like a coil spring. After implantation, the cannula would then pull itself under the skin of the back to store slack away from the animal's reach.

The apparatus for cross-perfusion was built around a special Teflon-lined, chemically inert four-way valve (No. 1, Type 4F4; Hamilton Co., Reno, Nevada). Two of the four female Luer connectors on the valve were joined to $5-\mathrm{ml}$ glass syringes, while the remaining two were connected to $25-\mathrm{cm}$ lengths of polyethylene tubing (P.E. 60). The free ends of these segments were fitted over one end of 2-cm sections of 21-ga hypodermic tubing which were used to connect the apparatus tubing to the cannulae for blood-mixing. The valve was designed such that blood could be drawn from two separate animals into the two syringes. With a turn of the valve handle, the blood could then be pumped back into the opposite animal from which it came. This valve and syringe system was located on a raised platform flanked by two Plexiglas restraint cages, all on a wooden base. These cages were triangular in cross-section $(10 \mathrm{~cm}$ on a side) and adjustable (maximum $21 \mathrm{~cm}$ long) by means of a sliding gate. For cross-perfusion, the animals were placed in these cages, their cannulae pulled up through open slits at the top, and then connected to the tubing leading from the valve and syringe system.

\section{Surgery}

The animals were prepared for surgery by doses of atropine sulfate and sodium pentobarbital $(35 \mathrm{mg} / \mathrm{kg})$. A $2-\mathrm{cm}$ incision was made over the right external jugular vein and blunt dissection was used to clear away connective tissue from approximately $1 \mathrm{~cm}$ of the vein at the point where it descends into the thorax. A small v-shaped cut was made in the vein and the Silastic tip of the cannula was threaded in until the flange junction between the silicone and polyethylene sections was about $3 \mathrm{~mm}$ inside the vein. A short length of silk was then tied around the cannulated vein just distal to the flange, thus securing the cannula in place with its tip in or near the vena cava of the heart. After the cannula was more securely anchored to the tissues of the neck, a sharpened steel tube (o.d. $=.035$ in.) was threaded beneath the skin from the incision, across the ventral surface of the neck, and around the left shoulder to emerge from between the scapulae. This tube was then used to draw the cannula through under the skin. Two or three loops of the coil-shaped portion of the cannula were allowed to retract themselves under the skin to be stored as slack. The cannula, which to this point was filled with a saline-heparin solution, was now filled with a viscous mixture ( 5 parts to 4 by weight) of heparin solution $(100 \mathrm{U} / \mathrm{ml})$ and polyvinylpyrolodone to help prevent blood from washing into the cannula tip and forming clots. The cannula was then pinched off near the skin and sealed with the heat of a match. After closure of the wound, the animal was given a prophylactic dose of penicillin $(50,000 \mathrm{U})$ and returned to its home cage.

\section{Procedure}

Briefly, the procedure involved the cross-perfusion of mildly deprived normal-weight animals (recipients) with obese, lean, or normal-weight partners (donors). Postperfusion food intake of the recipients was measured for evidence of changes related to the weight of the donor.

The 60 subjects were equally divided into three groups, designated obese, normal, and lean. Ten animals in the obese group were placed on the Corbit and Stellar (1964) high-fat diet $(2 / 3$ ground rat chow to $1 / 3$ vegetable oil) and soon reached weights $100 \mathrm{~g}$ in excess of their normal partners. Ten animals in the lean group were placed on reduced food rations for 5 days preceding the cannula-implant operations. A $10-12 \mathrm{~g}$ ration of chow per day was found to be sufficient to reduce and maintain these subjects at $80 \%$ control weight. All remaining animals continued to have constant food and water.

For 10 days prior to surgery, the 10 obese and 40 normal animals were placed on a 5 -h deprivation schedule beginning between 8:00 and 10:00 a.m. At this time, lean animals were given one-quarter of their daily ration so as to roughly place them on an equal basis with the other animals in regard to the size and timing of the last meal. After $5 \mathrm{~h}$ of deprivation, all of the normals were given $10 \mathrm{~g}$ of chow and left undisturbed for $30 \mathrm{~min}$. Paper trays were used to catch spillage, and careful measurements of intake were made. The obese animals had meanwhile been returned to their cages and food, while the lean animals were given the remaining three-quarters of their ration. At the end of the test period, normal animals were returned to constant food and water.

After 10 days on this regimen, a normal was paired with an obese, normal, or lean partner and the cannulae implanted. The pair was allowed 2 days for recovery, but were continued on the deprivation schedule. The polyvinylpyrolodone-heparin solution was changed twice daily (8:00 a.m. and 5:00 p.m.) at times remote from cross-perfusion in order to minimize possible drug effects.

Cross-perfusion was begun $4.5 \mathrm{~h}$ after deprivation and was followed immediately by the food intake test period (for the 10 recipients in each group). For the blood-mixing procedure, the viscous heparin mixture was withdrawn and the cannulae connected to the apparatus tubing. Two milliliters of blood were drawn from each animal at a rate of $2 \mathrm{ml} / \mathrm{min}$ and then infused into the opposite animal at a rate of $2-4 \mathrm{ml} / \mathrm{min}$. This procedure was repeated 13-15 times during the 30-min blood-mixing period. According to Davis and Miller (1966), this number of 2-ml transfusions throughout a 30 -min period is sufficient for a near-total mixing of blood supplies. At the completion of the blood-mixing, donor animals were returned to their cages where they received their normal diet or ration. Cross-perfusion was carried out for 4 consecutive days and then discontinued. Of the 30 experimental animals, 24 (8 in each group) were successfully cross-perfused with donors. Of these, 4 completed only three sessions and 1 completed only two sessions.

\section{RESULTS}

The food intake data are summarized by Figure 1. Four days of postperfusion intake by the normal rats after cross-perfusion with obese, normal, or lean partners is shown by the central two data points on each line. The other points on each line represent intake by the same animals on 2 days immediately preceding, and 2 days immediately succeeding crossperfusion. It is clear that food intake was reduced after blood-mixing and that this reduction is proportional to donor weight (see Table 1 for mean weights in each group). An analysis of variance on these data showed that food intake was significantly depressed, $\mathrm{F}(1,21)=27.5, \mathrm{p}<.001$, but more im- 
portantly showed a significant interaction between the perfusion-nonperfusion factor and the weight of the donor, $F(2,21)=3.5, p<.05$. While it appears that this interaction may be primarily due to the drop in intake by animals with obese partners, it nevertheless seems true that the heavier the donor is, the greater will be the reduction of the recipient's intake. An additional analysis of variance comparing nonperfusion test intake pre- and postoperatively showed that the surgery and daily heparin injections did not significantly reduce food intake, $F(1,21)=$ .41. To be sure that intake differences between the three groups were not due to differences in the weights of the recipient animals, intake was also plotted as a function of their metabolic weights (weight to the $3 / 4$ power). This provides a measure of an animal's intake relative to his metabolic needs

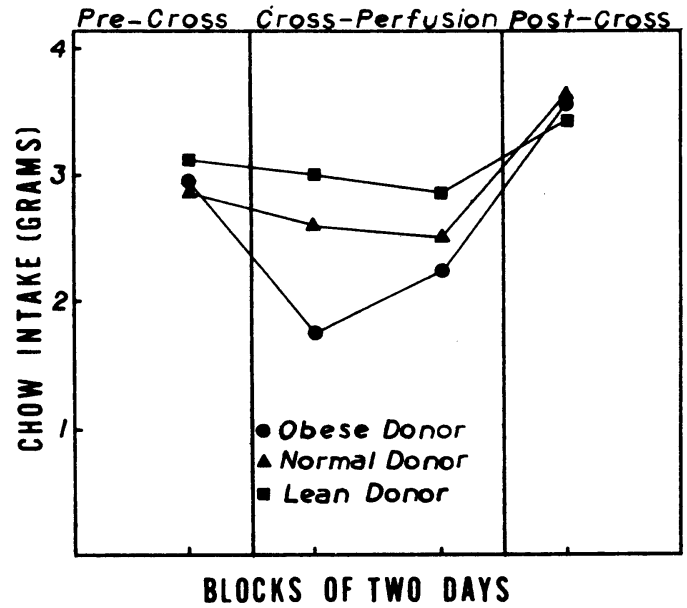

Figure 1. Mean 30-min chow intake of normal animals having obese, normal, or lean partners. Data points represent intake for 2 days prior to cross-perfusion, 4 days of cross-perfusion trials, and 2 days following these trials.

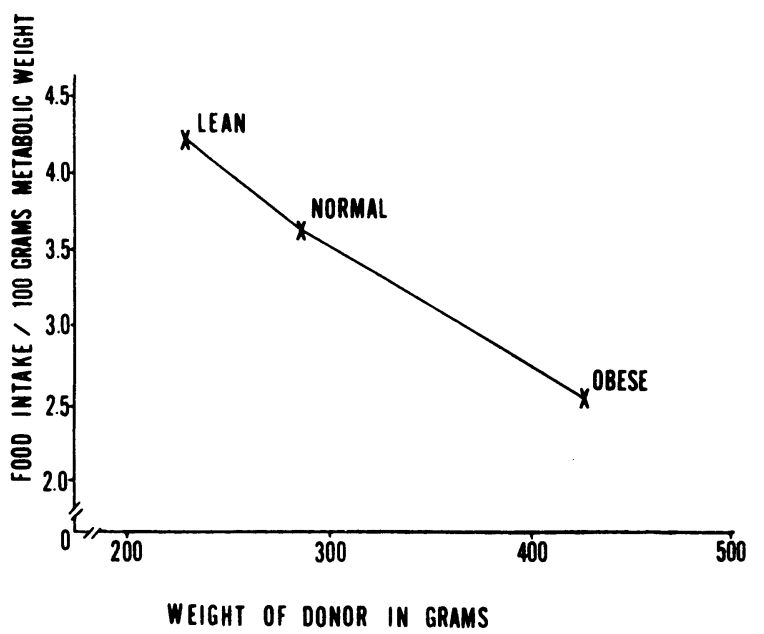

Figure 2. Mean 30-min chow intake of normal animals over 4 days of cross-perfusion with obese, normal, or lean partners. Intake is recorded as a function of the animal's metabolic weight (weight to the $3 / 4$ power).
Table 1

Mean Weight in Grams of Experimental Animals (Recipients) and Partners (Donors) in Different Groups

\begin{tabular}{lcl}
\hline Group & Recipients & Donors* \\
\hline Lean & 285 & $226(79)$ \\
Normal & 289 & $283(98)$ \\
Obese & 314 & $424(135)$ \\
\hline
\end{tabular}

*Numbers in parentheses indicate donor weight as a percentage of recipient weight.

(Kleiber, 1947). It can be seen from Figure 2 that there is nearly a linear relationship between food intake and donor weight.

\section{DISCUSSION}

Food intake of normal animals was reduced after cross-perfusion with partners having deficiencies or excesses of fat. If one considers the $25 \%$ reduction which occurred in the control (normal to normal) condition to represent only deleterious effects of blood-mixing, then the reductions of $40 \%$ and $14 \%$ in the obese and lean groups might be viewed as relative decreases or increases in intake attributable to the weight of the partner. These changes then might be explained by proposing that the recipient animals altered their intake in response to blood-bone "information" that they were in energy excess or deficit. The increase or decrease in intake was an effort to return their perceived weight to setpoint. Viewed in this fashion, these data provide support for the theory that a humoral signal, related to body adiposity, can influence food intake and thus regulate body weight.

Although total body fat was the most salient difference between the three groups (ascertained by visual postmortem examinations), the possibility must be entertained that dietary differences played a key role. The high-fat diet, especially, might have induced changes in plasma concentrations of free fatty acids (FFA) and glycerol (Bjorntorp, 1972). In an effort to minimize the effect of dietary factors, the animal room lights were set to come on at 4:00 a.m., $4 \mathrm{~h}$ prior to food deprivation. Since rats eat very little in the first hours of light (LeMagnen, Devos, Gaudilliere, Louis-Sylvestre, \& Tallon, 1973), there was then an effective deprivation period of $9 \mathrm{~h}$ before any blood-mixing. It was thought that this procedure would at least reduce the contribution of any short-term factors related to diet. It was for similar reasons that the lean animals were given part of their ration $5 \mathrm{~h}$ before cross-perfusion. This was thought to mimic, in the lean animals, the short-term nutritional state of the obese and normal animals-that is, having a small amount of food in the stomach prior to deprivation. Although these procedures may not have totally eliminated shortterm factors, the data strongly suggest that a chemical signal, related to body fat, is present in 
the blood and is capable of having excitatory or inhibitory effects on food intake.

With regard to the exact nature of this signal, little is known. Nisbett's (1972) suggestion that free fatty acids (FFA), mobilized from the adipose tissues, function as a lipostatic feedback agent is supported by the finding that rats have a diurnal cycle of fat mobilization and deposition (LeMagnen et al., 1973). Since in this experiment all animals were fooddeprived, a condition which induces elevated FFA levels (Kennedy, 1966) and were maintained on heparin, a drug having ketogenic properties (Seoane, Warner, \& Seoane, 1972), it is unlikely that FFA could have been the factor differentiating the three groups. Other suggestions, such as the one by Woods, Decke, and Vasselli (1974) that the ratio of insulin to growth hormone is the relevant humoral signal, cannot be answered with these data and the resolution of this issue must await further research.

How does this signal, whatever its nature, affect total food intake? While this question may require a host of explanations, one interesting possibility is that the effects of the humoral factor are mediated through taste. Stating the case directly, Cabanac (1971) has proposed that a central "ponderostat" detects deviations from setpoint weight and alters food intake by rendering the diet more or less palatable. This position is supported by work showing that animals with elevated weights tend to find food less palatable (Sclafani \& Kluge, 1974), while underweight animals are more responsive to good tasting food than normals (Jacobs \& Sharma, 1969). It does not seem unreasonable to propose that a humoral factor, lipostatic in nature, might be responsible for these changes in taste, and hence food intake. Although tenuous and unsubstantiated, this is one possible explanation of how a humoral factor could affect food intake without interfering with the shortterm needs of the animal. The animal would still consume a large or small amount depending on its short-term requirements. However, this amount would be further increased or decreased (by means of palatability) depending on whether the animal was above or below setpoint.

Throughout this discussion, the assumption has been made that there may be one mechanism controlling short-term "caloric" eating and another governing long-term "regulatory" eating. With the growing appreciation that food intake regulation is a multifaceted and very complex phenomenon, it should be remembered that such distinctions are our own and may not be present in the living organism. There is now evidence that both short-term satiety and long-term weight changes can modify food intake through humoral mediators. Whether these are distinct phenomena or simply different dimensions of the same underlying regulatory process is an important question for further research.

\section{REFERENCES}

BJORNTORP, P. Disturbances in the regulation of food intake. Advances in Psychosomatic Medicine, 1972, 7, 116-147.

CabanaC, M. The physiological role of pleasure. Science, 1971, 173, 1103-1107.

Corbitt, J. D., \& Stellar, E. Palatability, food intake, and obesity in normal and hyperphagic rats. Journal of Comparative and Physiological Psychology, 1964, 58, 63-67.

Davis, J. D., Gallagher, R. J., \& LaDove, R. Food intake controlled by a blood factor. Science, 1967, 156, 1247-1248.

Davis, J. D., Gallagher, R. J., Ladove, R. F., \& Turausky, A. J. Inhibition of food intake by a humoral factor. Journal of Comparative and Physiological Psychology, 1969, 67, 407-414.

DAvis, J. D., \& Miller, N. E. A technique for mixing the blood of unanesthetized rats. Journal of Applied Physiology, 1966, 21, 1873-1874.

HAN, P. W., Mu, J. Y., \& LePkovsky, S. Food intake of parabiotic rats. American Journal of Physiology, 1963, 205, 1139-1143.

HeRVEY, G. R. The effects of lesions in the hypothalamus in parabiotic rats. Journal of Physiology, London, 1959, 145, 336-352.

Hoebel, B. G., \& Teitelbaum, P. Weight regulation in normal and hypothalamic hyperphagic rats. Journal of Comparative and Physiological Psychology, 1966, 61, 189-193.

Jacobs, H. L., \& Sharma, K. N. Taste versus calories: Sensory and metabolic signals in the control of food intake. Annals of the New York Academy of Sciences, 1969, 157, 1084-1112.

KENNEDY, G. C. The role of depot fat in the hypothalamic control of food intake in the rat. Proceedings of the Royal Society, London (Series B), 1953, 140, 578-592.

KENNEDy, G. C. Food intake, energy balance, and growth. British Medical Bulletin, 1966, 22, 216-220.

KLeIBer, M. Body size and metabolic rate. Physiological Reviews, 1947, 27, 511-541.

LeMagnen, J., Devos, J., Gaudilliere, J. P., Louis-Sylvestre, J., \& TAllon, S. Role of a lipostatic mechanism in regulation by feeding of energy balance in rats. Journal of Comparative and Physiological Psychology, 1973, 84, 1-23.

Liebelt, R. A., Bordelon, C. B., \& Liebelt, A. G. The adipose tissue system and food intake. In E. Stellar \& J. M. Sprague (Eds.), Progress in physiological psychology. New York: Academic Press, 1973. Pp. 211-252.

MAYER, J. Glucostatic mechanism of regulation of food intake. New England Journal of Medicine, 1953, 249, 13-16.

NisBeTt, R. E. Hunger, obesity, and the ventromedial hypothalamus. Psychological Review, 1972, 79, 433-453.

Powley, T. L., \& KeEsey, R. E. Relationship of body weight to the lateral hypothalamic feeding syndrone. Journal of Comparative and Physiological Psychology, 1970, 70, 25-36.

Sclafani, A., \& Kluge, L. Food motivation and body weight levels in hypothalamic hyperphagic rats: A dual lipostatic model of hunger and appetite. Journal of Comparative and Physiological Psychology, 1974, 86, 28-46.

Seoane, J. R., Baile, C. A., \& Martin, F. H. Humoral factors modifying feeding behavior of sheep. Physiology and Behavior, 1972, 8, 993-995.

Seoane, J. R., Warner, R. G., \& Seoane, N. A. Heparin-induced lipolysis and feeding behavior in sheep. Physiology and Behavior, 1972, 9, 419-422.

W Alike, B. C., \& Smith, O. A. Regulation of food intake during intermittent and continuous cross-circulation in monkeys (Macaca mulatta). Journal of Comparative and Physiological Psychology, 1972, 80, 372-381.

Woods, S. C., DeCke, E., \& VAsselli, J. R. Metabolic hormones and regulation of body weight. Psychological Review, 1974, 81, 26-43.

(Received for publication May 17, 1976; revision received June 24,1976 .) 